Discrete Comput Geom 34:143-166 (2005)

DOI: $10.1007 / \mathrm{s} 00454-005-1157-3$

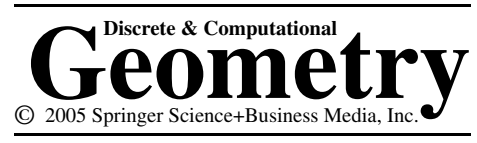

\title{
Hyperbolic Regular Polygons with Notched Edges
}

\author{
Casey Mann \\ HPR 102, University of Texas at Tyler, \\ 3900 University Blvd., Tyler, TX 75799, USA \\ cmann@uttyler.edu
}

\begin{abstract}
In this article we solve the tiling problem for hyperbolic and Euclidean regular polygons whose edges are notched with matching bumps and nicks.
\end{abstract}

\section{Introduction}

One of the biggest, if not the biggest problems in tiling theory is the tiling problem, which asks if one can always decide in some algorithmic manner, when given an arbitrary collection of tiles (i.e., a protoset), whether or not the given protoset tessellates the plane. More precisely, the tiling problem for a class $\mathcal{T}$ of protosets of tiles asks if there exists an algorithm or standard procedure that can decide in a finite number of steps if an arbitrary protoset $P \in \mathcal{T}$ tessellates the plane or not. If such an algorithm exists for $\mathcal{T}$, then the tiling problem is said to be decidable for $\mathcal{T}$; otherwise, it is said to be undecidable for $\mathcal{T}$. This problem is at the center of many open problems in tiling theory, and its complete solution would cast light in many directions. Some well-known examples of related open problems are the Einstein problem, which asks if there exists an aperiodic monotile, and Heesch's tiling problem, which we describe later. The tiling problem can be stated in many settings and can be restricted to consider only certain kinds of protosets. It was shown by Berger that if $\mathcal{T}$ is the class of all protosets of planar tiles, then the tiling problem is undecidable for $\mathcal{T}$ [1]. On the other hand, we may find nontrivial classes of protosets for which the tiling problem is decidable: one such example is the class of protosets that contain only a single convex planar hexagon [3]. Surprisingly, for the class of protosets that contain a single convex planar pentagon, the tiling problem has not been solved. In this paper we discuss the tiling problem for a certain class of tiles which for the most part lie in the hyperbolic plane. These tiles will all have matching conditions on each of their edges which we call bumps and nicks (as in Fig. 2). 


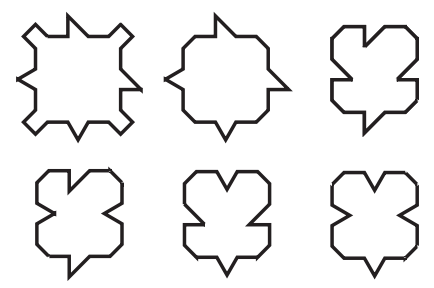

Fig. 1. Robinson's aperiodic protoset.

To motivate the interest in tiles formed from notched polygons (a special kind of which are the focus of this paper), we point out that historically and very recently these kinds of tiles have been a rich source of examples for various famous tiling problems [2], including the Einstein problem, Heesch's tiling problem, and the problem of isohedral numbers. A few examples of this are the following: One of the very first aperiodic tile protosets, discovered by Robinson [8], contained six polygons with edges marked with nicks and bumps (Fig. 1). Later, Penrose discovered an aperiodic protoset containing only two tiles, and these tiles can be seen as rhombi with notched edges [7]. Ammann has gotten considerable milage out of tiles whose edges are marked with bumps and nicks; first with his aperiodic protoset and second with his Heesch number 3 example (Fig. 3) [3]. More recently tiles with Heesch numbers 4 and 5 have been discovered, and they too are formed from polygons with notched edges [4]. Unpublished examples with high isohedral numbers have been recently discovered, and some of these are formed from notched polygons.

In the literature there are a several papers of particular relevance to the work presented here. These papers fall into two categories: those involving aperiodic protosets in $\mathbb{H}^{2}$ and those involving Heesch's tiling problem. Penrose in [7] gives an example of a notched polygon that is a weakly aperiodic tile in $\mathbb{H}^{2}$. In [6] tessellations of $\mathbb{H}^{2}$ with convex, but not regular, polygons are discussed. In particular, the existence of weakly aperiodic polygons in $\mathbb{H}^{2}$ is proven. A key idea in both of these papers is that tiles with a certain imbalance (such as a tile with more bumps than nicks) cannot tessellate a compact quotient of $\mathbb{H}^{2}$, and are hence at least weakly aperiodic. This observation applies to most of the tiles that are discussed in this article; that is, most of the tiles we discuss here are weakly aperiodic as well, but none are strongly aperiodic. ${ }^{1}$ These combinatorial imbalances also play a role in deciding if a given marked tile can tessellate the plane or not - this is where Heesch's tiling problem comes into play.

Loosely stated, Heesch's tiling problem asks for which nonnegative integers $N$ do there exist a planar tile $T$ that can be used to form a partial tessellation consisting of $N$ layers, but not $N+1$ layers, of congruent copies of $T$ surrounding a centrally placed copy of $T$. This integer $N$ is called the Heesch number of the tile and is denoted by $H(T)$.

\footnotetext{
${ }^{1}$ A protoset $\mathcal{P}$ is said to be strongly aperiodic if for every tessellation admitted by $\mathcal{P}$ the corresponding symmetry group of the tessellation contains no infinite cyclic subgroups. If the symmetry group of every tessellation admitted by $\mathcal{P}$ contains at least one infinite cyclic subgroup, but no two "nonparallel" infinite cyclic subgroups, then $\mathcal{P}$ is said to be weakly aperiodic.
} 

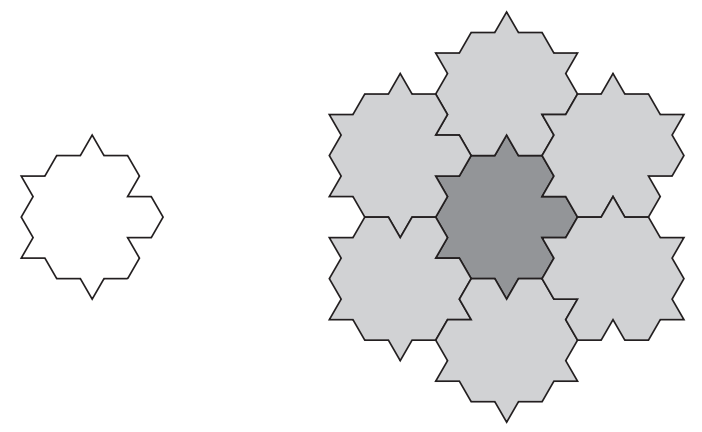

Fig. 2. A Heesch number 1 monotile. A second layer cannot be formed.

Tiles with a finite Heesch number cannot tessellate the plane, but can tessellate outward some number of layers. Of particular interest is the question of whether or not there exists a maximum possible Heesch number over the set of all possible planar tiles. Heesch's tiling problem has been solved for $0 \leq N \leq 5$, but in general Heesch's tiling problem is unsolved [4], [5]. Several of the tiles that serve as solutions to Heesch's problem for particular values of $N$ are tiles whose edges are marked with bumps and nicks: the tile of Fig. 2 is a solution to Heesch's tiling problem with $N=1$ and the tile of Fig. 3 is a solution for $N=3$. Of course, Heesch's tiling problem can be posed for tiles in $\mathbb{H}^{2}$, and we will be considering the Heesch numbers of tiles in $\mathbb{E}^{2}$ and $\mathbb{H}^{2}$.

We now give some terminology, basic facts, and assumptions for the rest of this paper. First, we remind the reader that in $\mathbb{H}^{2}$ there are infinitely many regular polygons that tessellate $\mathbb{H}^{2}$ vertex-to-vertex. In fact, for every pair of positive integers $n$ and $q$ satisfying $1 / n+1 / q<\frac{1}{2}$ there is a hyperbolic regular $n$-gon with interior vertex angle $2 \pi / q$ that tessellates $\mathbb{H}^{2}$ [9] in a unique way (up to symmetry). A regular $n$-gon with interior vertex angle $2 \pi / q$ must necessarily tile $\mathbb{H}^{2}$ in such a way that $q$ copies of the $n$-gon meet at every

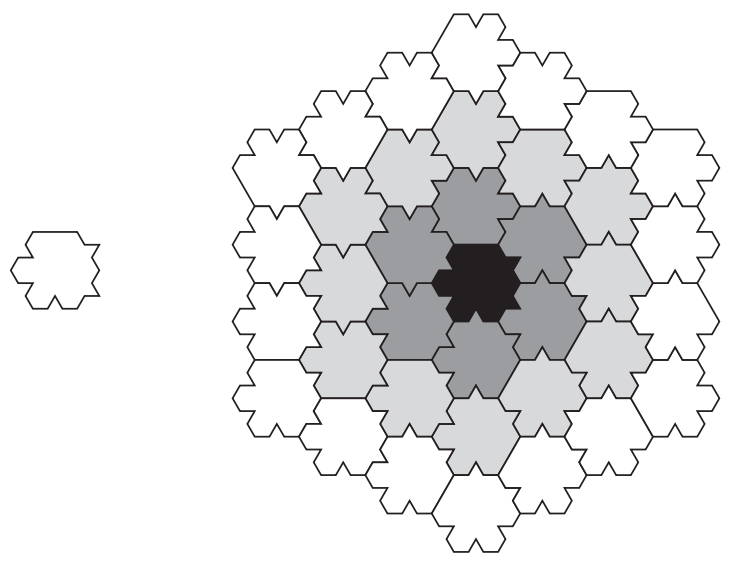

Fig. 3. Ammann's Heesch number 3 monotile. 


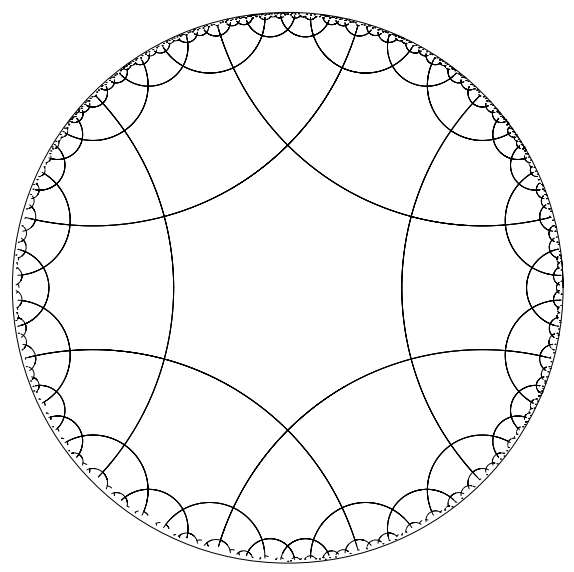

Fig. 4. A tiling of $\mathbb{H}^{2}$ by $(6,4)$-gons.

vertex. In Fig. 4 we see a tiling of $\mathbb{H}^{2}$ by hexagons with interior vertex angle $\pi / 2(q=4)$. It may also be noted that if $n$ and $q$ are positive integers satisfying $1 / n+1 / q=\frac{1}{2}$, then there is a regular polygon in $\mathbb{E}^{2}$ with $n$ edges and vertex angle $2 \pi / q$. One checks that there are only three such pairs- $(3,6),(4,4)$, and $(6,3)$ corresponding to the equilateral triangle, the square, and the regular hexagon, respectively, and these shapes tessellate $\mathbb{E}^{2}$. We call a regular $n$-gon in either $\mathbb{H}^{2}$ or $\mathbb{E}^{2}$ with vertex angle $2 \pi / q$ an $(n, q)$-gon. We also note that if the pair $(n, q)$ satisfies $1 / n+1 / q>\frac{1}{2}$, then there is a spherical $(n, q)$-gon with $n$ sides with interior vertex angle $2 \pi / q$, but we do not concern ourselves with spherical tiles here.

As can be seen in Fig. 4, the number of polygons grows rapidly as the number of coronas increases. The precise number of $(n, q)$-gons in $k$ coronas is given in the following lemma [4].

Lemma 1.1. Let $\mathcal{F}_{(n, q)}(k)$ be the number of $(n, q)$-gons in a configuration of $k$ coronas. Then

$$
\mathcal{F}_{(n, q)}(k)=\frac{2^{-(1+k)}\left(2^{2+k} q x+n^{2}(-2+q)^{2}(y-z)-n(-2+q)(2 q(y-z)+x(y+z))\right)}{(2 n+2 q-n q) x},
$$

where

$$
\begin{aligned}
& x=\sqrt{-4+(2+n(-2+q)-2 q)^{2}}, \\
& y=(2+n(-2+q)-2 q-x)^{k},
\end{aligned}
$$

and

$$
z=(2+n(-2+q)-2 q+x) x^{k}
$$

Throughout this paper we will be examining coronal configurations of $(n, q)$-gons whose edges are marked with matching rules in a certain way. To show that a given 


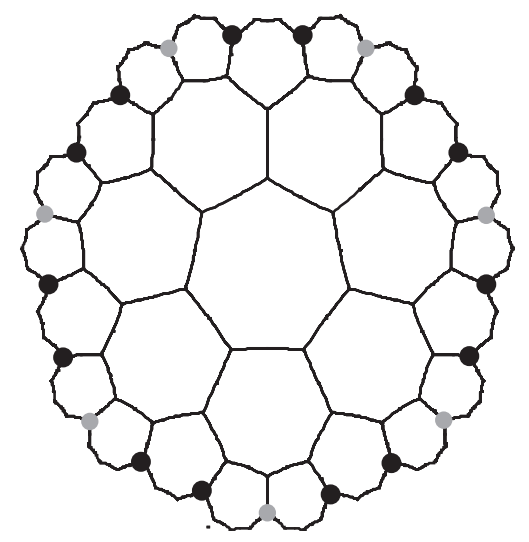

Fig. 5. The boundary vertices of a coronal configuration.

marked $(n, q)$-gon tiles $\mathbb{H}^{2}$, we invariably proceed inductively by showing that one can "shell out" and form arbitrarily many layers, implying that a tiling by the marked $(n, q)$ gon exists. Since we will be forming layers inductively, it is important to understand what is happening on the boundary of any coronal configuration consisting of one or more layers around a centrally placed copy of the tile. First notice that every vertex on the boundary of a coronal configuration is incident to either two edges or three edges, depending on whether or not the vertex is incident to an edge shared by two abutting tiles in the outermost corona (Fig. 5). To form another corona, each of these vertices will be surrounded by tiles. Since $q \geq 3$ for the hyperbolic tiles of interest, this means that any tile that is placed in the next corona will share at most two edges with tiles of the previous corona. We use this fact throughout.

Now consider $(n, q)$-gons each of whose edges is notched either inward or outward so that all of the notches are the same shape. To simplify our notation later, we equivalently require that the edges of our $(n, q)$-gons are each marked by either a zero $(0)$ or a one (1), and for fixed $n$ and $q$, two $(n, q)$-gons may meet vertex-to-vertex along their edges if the abutting edges' markings sum to 1, as in Fig. 6.

It must be stressed here that in the formation of tessellations with these polygons, we allow the use of both orientation-preserving and orientation-reversing isometries. If $T$ is an $(n, q)$-gon, the markings on $\partial T$ (the boundary of $T$ ) will be written as a finite sequence in 0 and 1. For example, the boundary of the tile in Fig. 2 is represented by 111100. Two such sequences correspond to equivalent $(n, q)$-gons (up to isometry) if the sequences are cyclic permutations and/or reverse orderings of one another (where

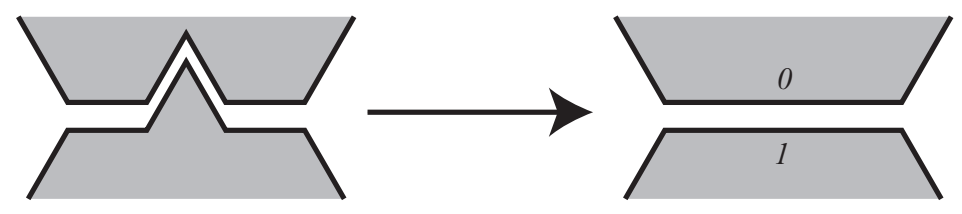

Fig. 6. All edges are notched with bumps and nicks of the same shape. 
cyclic permutation corresponds to rotation of the original tile and reversing the order corresponds to reflection of the tile). For example, a $(6,4)$-gon with boundary edges marked 101001 is equivalent to one marked 110100 or one marked 100101.

With our $(n, q)$-gons marked as previously described, we classify which finite boundary sequences in 0 and 1 give rise to tessellations of the plane and those that do not. In Section 2 we begin by solving the tiling problem for the class of $(n, q)$-gons with $q \geq 4$ (Theorem 2.1), then in Section 3 we solve the case $q=3$ separately (Theorem 3.1). In both Sections 2 and 3, for the notched polygons that do not tessellate the plane, we are interested in the Heesch numbers of such tiles. In Section 4 we indicate some open problems and possible future directions for research.

\section{2. $(n, q)$-Gons with $q \geq 4$}

The goal for this section is to prove the following theorem which classifies the $(n, q)$ gons with $q \geq 4$ whose edges are all marked with a 0 or a 1 that can tessellate the plane:

Theorem 2.1. Let $T$ be an $(n, q)$-gon with $q \geq 4$ such that every boundary edge of $T$ is marked either 0 or 1 .

(i) If $n$ is odd, then $T$ tessellates if and only if the equence of markings on $\partial T$ contains both 00 and 11 .

(ii) If $n$ is even, then $T$ tessellates if and only if either the sequence of markings on $\partial T$ contains 00 and 11 or if the sequence of markings on $\partial T$ alternates in 0 and 1.

(iii) If $T$ does not tessellate, then $H(T)=0$.

So, as you can see, the case $q \geq 4$ is remarkably simple. To prove Theorem 2.1, we need to establish a few lemmas:

Lemma 2.1. Let $T$ be an $(n, q)$-gon such that every edge on $\partial T$ is marked with a 0 or a 1. If $T$ tessellates, then the sequence or markings on $\partial T$ must contain at least two 0 's and two 1's. Moreover, if the sequence of markings on $\partial T$ contains only one 0 or no 0 's (or if the sequence of markings on $\partial T$ contains only one 1 or no 1 's), then $H(T)=0$.

Proof. It is obvious that if the sequence of markings on $\partial T$ contains nothing but 0 's on the boundary, then $T$ will not tessellate and $H(T)=0$. If the sequence of markings on $\partial T$ contains all 0 's and only a single 1 , then $\partial T$ must contain 00 since the number of edges must be greater than or equal to 3 . We then see that we cannot fill in tiles around a vertex between edges marked 00, because a 11 will be needed to fill in tiles around the vertex between consecutive edges marked 0 (see Fig. 10). Again, a first corona cannot be formed, so $H(T)=0$.

Lemma 2.2. Let $T$ be an $(n, q)$-gon where $q \geq 4$ and every edge is marked either 0 or 1 . If the sequence of marking on $\partial T$ contains both 00 and 11 , then $T$ tessellates. 


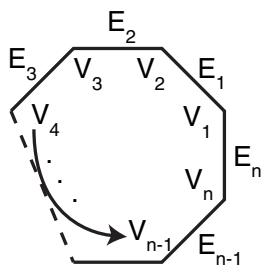

Fig. 7. The $(n, q)$-gon of Lemma 2.2.

Proof. Since the sequence of markings on $\partial T$ contains 00 , then it must also contain 001 or 100. Likewise, since $\partial T$ contains 11 , then it must also contain 011 or 110 . Additionally $\partial T$ contains 10 or 01 . We now give an algorithm requiring only these combinations to ensure that $T$ tessellates:

Step 1. Start with a single tile with vertices $V_{1}, V_{2}, \ldots, V_{n}$ and edges $E_{1}, E_{2}, \ldots, E_{n}$ (Fig. 7). ${ }^{2}$ Around each vertex $V_{k}$ we first fill in $q-3$ tiles as shown in Fig. 8, leaving the "slots" adjacent to the edges $E_{k}$ empty. The only edge combination needed to do this is 10 , which is contained in $\partial T$ or in the boundary of a reflected copy of $T$.

Step 2. Referring to Fig. 8, we fill in the tiles that will be adjacent to the edges $E_{k}$. Notice that the edge immediately to the left of $E_{k}$ is marked 0 and the edge immediately to the right of $E_{k}$ is marked 1. Depending on whether $E_{k}$ is marked 0 or 1, we will need an edge combination of 100 or 110 , respectively, to fill in the space adjacent to edge $E_{k}$. However, these edge combinations are contained in either $\partial T$ or in the boundary of a reflected copy of $T$. In this way we complete the entire first corona.

To complete every additional corona, we repeat steps 1 and 2 on coronal configurations. The only difference will come at vertices on the boundary of the configuration which are incident to three edges, where instead of filling in $q-3$ vertices in step 1, we fill in $q-4$ vertices instead. In the case that $q=4$ we must specify a different manner for filling in tiles around valence-3 vertices on the boundary. Referring to Fig. 9, it does not matter what the edge markings are for the edges incident to the valence- 3 vertex-we see that the edge combinations 001 and 011 are the only combinations needed.

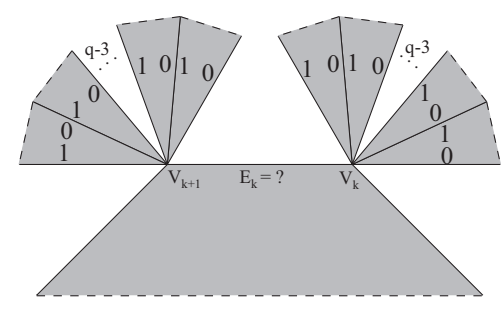

Fig. 8. Filling around a vertex.

\footnotetext{
${ }^{2}$ Many of the figures in this paper are not meant to be geometrically accurate depictions of configurations of tiles in $\mathbb{H}^{2}$; rather, the figures are intended to depict accurately the combinatorial structure of local configurations of tiles in $\mathbb{H}^{2}$.
} 


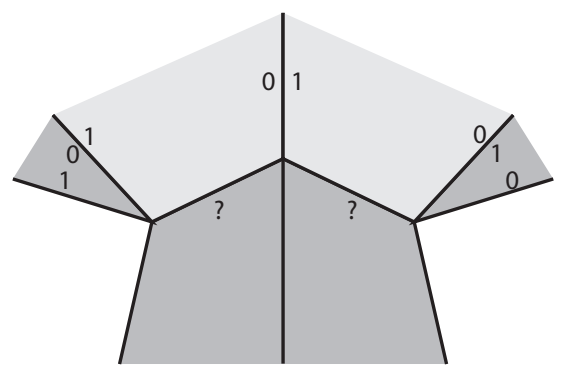

Fig. 9. Filling around a valence- 3 vertex when $q=4$.

Geometrically, our tiles are no different from the regular $(n, q)$-gons which are known to tessellate $\mathbb{H}^{2}$ exactly in the vertex-to-vertex manner we have described. Therefore this algorithm gives a tessellation of $\mathbb{H}^{2}$.

Lemma 2.3. Let $T$ be an $(n, q)$-gon such that every boundary edge of $T$ is marked either 0 or 1 . If $\partial T$ contains 00 but not 11 (or contains 11 but not 00 ), then $T$ does not tessellate and $H(T)=0$.

Proof. In forming a first corona, start trying to fill in tiles around the vertex between the edges marked 00 in a counterclockwise manner starting from the rightmost edge marked 0 (Fig. 10). In order to place the last tile adjacent to the leftmost edge marked 0 , you will need the edge combination 11, which is not present.

Lemma 2.4. Let $T$ be a $(2 n, q)$-gon where the sequence of markings on $\partial T$ is alternating in 0 and 1 . Then $T$ tessellates.

Proof. Just note that the boundary of any configuration formed from copies of such a tile must alternate in 0 and 1 since the edge markings emanating from a vertex incident to two or more tiles must have opposite parity. So coronas of all sizes can be formed and a tessellation by this tile must exist.

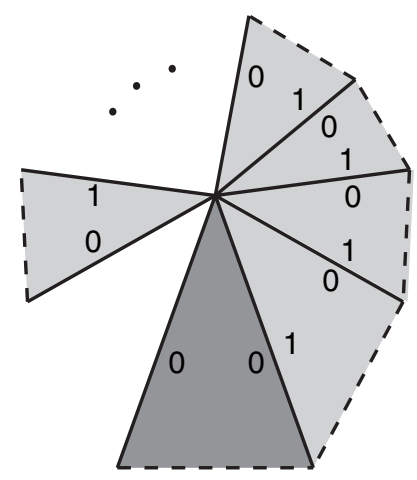

Fig. 10. Lemma 2.3: without a 11 in $\partial T$, we cannot fill around a 00 . 
Now we combine the previous lemmas to give a proof of Theorem 2.1:

Proof of Theorem 2.1. (i) Assume that the boundary our $(n, q)$-gon contains at least two edges marked 0 and two edges marked 1 (since by Lemma 2.1 at least this much is needed for it to have a hope of tessellation). If $T$ is any such $(n, q)$-gon with $n$ odd, then $\partial T$ must contain either 00 or 11 for parity reasons. Lemma 2.2 says that if $\partial T$ contains both 00 and 11, then $T$ tessellates. So if $\partial T$ does not contain 00 , then $\partial T$ must contain 11, and so by Lemma 2.3, $T$ does not tessellate. Likewise, if $\partial T$ does not contain 11, then $\partial T$ must contain 00 , and so in this case $T$ does not tessellate. Therefore, $T$ will tessellate exactly when $\partial T$ contains both 00 and 11 .

(ii) Suppose $T$ is any $(n, q)$-gon with $n$ even. If $\partial T$ does not contain 00, then $\partial T$ is either alternating in 0 and 1 or $\partial T$ contains 11 . If $\partial T$ is alternating in 0 and 1 , then $T$ tessellates by Lemma 2.4. If $\partial T$ contains 11, then $T$ does not tessellate by Lemma 2.3 . Likewise if $\partial T$ does not contain 11, then $\partial T$ either alternates in 1 and 0 or $\partial T$ contains 00 . So the $(n, q)$-gons with $n$ even which tessellate are exactly those whose boundary alternates in 0 and 1 or those whose boundary contains both 00 and 11 .

(iii) The only tiles from parts (i) and (ii) which do not tessellate are those guaranteed not to tessellate by Lemmas 2.1 and 2.2, and so they have Heesch number 0 .

\section{3. (n, 3)-Gons}

Unfortunately, Lemma 2.2 will not work for $(n, 3)$-gons because it does not account for the valence- 3 vertices on the boundaries of coronal configurations. To place a third tile at a valence- 3 vertex on the boundary of a configuration requires the newly placed tile to meet two existing tiles simultaneously, rendering the algorithm ineffective. Therefore, a new approach is needed in the valence- 3 case.

Our goal here is to lay out a classification theorem for $(n, 3)$-gons whose edges are marked 0 or 1 as before. Our approach will be to classify these tiles by which triples of 0 and 1 they contain (up to reflection). To see where we are going with this, consider the following examples:

- $\partial T=01010101$. The only triples of 0 and 1 that this sequence contains are 010 and 101. So $T$ is a member of the class of tiles whose boundaries contain and only contain the triples 010 and 010 . In fact, any tile whose boundary alternates in 0 and 1 is a member of this class of tiles.

- $\partial T=11001100$. The triples of 0 and 1 that this sequence contains are 110, 100, 001, and 011 . However, recall that we are allowing orientation-reversion isometries to be allowed in the tessellation process, so if $\partial T$ contains 110 , then we can also "flip" $T$ to get the triple 011 . So we think of 110 and 011 as being interchangeable. Likewise, 100 and 001 are interchangeable. Therefore, the only triples contained in $\partial T$ are 110 and 001. In fact, any member of the class of tiles whose boundaries contain and only contain the triples 110 and 001 must have a boundary that alternates in 00 and 11.

- Consider the class of tiles whose boundaries contain and only contain the triples 111 and 001 . Notice that if $\partial T$ contains 111 and at least one 0 , then $\partial T$ must also contain 110 . Therefore, the class of tiles whose boundaries contain only the triples 111 and 001 is a null class. 
- Consider a tile $T$ with $\partial T=101100010$. What triples does $\partial T$ contain? $\partial T$ contains 110,101, 010, 001, and 000. So $T$ belongs to the class of tiles whose boundaries contain and only contain these five triples of 0 and 1 . Another member of this class is any tile marked 01101011000.

There are only six possible triples of 0 and 1 (modulo the equivalence we described before): 000, 001, 010, 011, 101, and 111. Therefore, we can make a fairly short classification of tiles based on which of these triples a particular tile's boundary sequences contains and only contains. Tables 1-6 list all of the possible 63 tile classes based on combinations of triples of 0 and 1 that may appear in the boundary sequences.

Table 1. Classes of tiles whose boundary sequences contain and only contain one triple.

\begin{tabular}{l|l|l|l|l|l|}
111 & 110 & 101 & 010 & 001 & 000 \\
\hline
\end{tabular}

Table 2. Classes of tiles whose boundary sequences contain and only contain two triples.

\begin{tabular}{|l|l|l|l|l|l|l|l|}
\hline 111 & 111 & 111 & 111 & 111 & 011 & 011 & 011 \\
011 & 101 & 010 & 001 & 000 & 101 & 010 & 001 \\
\hline 011 & 101 & 101 & 101 & 010 & 010 & 001 & \\
000 & 010 & 001 & 000 & 001 & 000 & 000 & \\
\hline
\end{tabular}

Table 3. Classes of tiles whose boundary sequences contain and only contain three triples.

\begin{tabular}{|l|l|l|l|l|l|l|l|l|l|}
\hline 111 & 111 & 111 & 111 & 111 & 111 & 111 & 111 & 111 & 111 \\
011 & 011 & 011 & 011 & 101 & 101 & 101 & 010 & 010 & 001 \\
101 & 010 & 001 & 000 & 010 & 001 & 000 & 001 & 000 & 000 \\
\hline 011 & 011 & 011 & 011 & 011 & 011 & 101 & 101 & 101 & 010 \\
101 & 101 & 101 & 010 & 010 & 001 & 010 & 010 & 001 & 001 \\
010 & 001 & 000 & 001 & 000 & 000 & 001 & 000 & 000 & 000 \\
\hline
\end{tabular}

Table 4. Classes of tiles whose boundary sequences contain and only contain four triples.

\begin{tabular}{|l|l|l|l|l|l|l|l|}
\hline 111 & 111 & 111 & 111 & 111 & 111 & 111 & 111 \\
011 & 011 & 011 & 011 & 011 & 011 & 101 & 101 \\
101 & 101 & 101 & 010 & 010 & 001 & 010 & 010 \\
010 & 001 & 000 & 001 & 000 & 000 & 001 & 000 \\
\hline 111 & 111 & 011 & 011 & 011 & 011 & 101 & \\
101 & 010 & 101 & 101 & 101 & 010 & 010 & \\
001 & 001 & 010 & 010 & 001 & 001 & 001 & \\
000 & 000 & 001 & 000 & 000 & 000 & 000 & \\
\hline
\end{tabular}

Table 5. Classes of tiles whose boundary sequences contain and only contain five triples.

\begin{tabular}{|l|l|l|l|l|l|}
\hline 111 & 111 & 111 & 111 & 111 & 011 \\
011 & 011 & 011 & 011 & 101 & 101 \\
101 & 101 & 101 & 010 & 010 & 010 \\
010 & 010 & 001 & 001 & 001 & 001 \\
001 & 000 & 000 & 000 & 000 & 000 \\
\hline
\end{tabular}


Table 6. The only class of tiles whose boundary sequences contain and only contain six triples.

\begin{tabular}{l|}
111 \\
011 \\
101 \\
010 \\
001 \\
000 \\
\hline
\end{tabular}

At this point we wish to remove any entries in Tables 1-5 that are empty or that we can immediately identify as nontilers with Heesch number 0 . We make the combinatorial observation about all classes containing both 0 and 1 . If 111 is present, then 011 must be present, and if 000 is present, then 001 must be present. Several classes do not satisfy this condition and are therefore empty classes. For similar combinatorial reasons, classes containing 010 must contain either 001 or 101, and classes containing 101 must also contain either 010 or 011 , and we eliminate all empty classes not satisfying these criteria. Additionally, we can apply Lemma 2.3 to the elimination process and remove those entries that allow 00 but not 11 and vice versa since such tiles we know must have Heesch number 0 . Finally, note that some of the pairs of classes are dual (i.e., interchanging 0 and 1 in one class results in the other). We only consider one representative class from each dual pair as they are combinatorially equivalent (though not geometrically equivalent). After making these eliminations, we arrive at the viable classes in Tables 7 and 8 . Theorem 3.1 classifies these classes as either tiling or nontiling:

Theorem 3.1. Let $T$ be any (n,3)-gon in $\mathbb{H}^{2}$ or $\mathbb{E}^{2}$ such that every edge of $\partial T$ is marked with a 0 or a 1 .

1. If $T$ belongs to one of the classes in Table 7 or a dual to one of these classes, then $T$ tessellates.

2. If $T$ belongs to one of the classes in Table 8 or a dual to one of these classes, then $T$ does not tessellate. Moreover, any tile $T$ belonging to one of these classes has $H(T)=1$.

3. Members of any other nonempty class have Heesch number 0 .

Table 7. The tessellating classes (up to duality).

\begin{tabular}{|l|l|l|l|l|l|l|l|}
\hline & & 111 & 111 & 011 & 111 & 111 & 111 \\
101 & 011 & 011 & 011 & 101 & 011 & 011 & 011 \\
010 & 001 & 010 & 001 & 010 & 101 & 010 & 101 \\
& & 001 & 000 & 001 & 010 & 001 & 010 \\
& & & & & 001 & 000 & 001 \\
\hline
\end{tabular}

Table 8. The nontessellating classes (up to duality) with Heesch number 1.

\begin{tabular}{|l|l|l|}
\hline 111 & 011 & 111 \\
011 & 101 & 011 \\
001 & 001 & 101 \\
& 001 \\
\hline
\end{tabular}


Proof. Part 3 was proven in our discussion of eliminating classes from Tables 1-6. We handle each of the class in Tables 7 and 8 as a separate case. We enumerate the cases in the following manner: the entries in Table 7 are enumerated from left to right as $\mathcal{T}_{1}-\mathcal{T}_{8}$ and the entries in Table 8 are enumerated as $\mathcal{N}_{1}, \mathcal{N}_{2}$, and $\mathcal{N}_{3}$.

Class $\mathcal{T}_{1}$. Let $T$ be any $(n, q)$-gon belonging to this class. Then we must have $n$ even and $\partial T$ alternating in 0 and 1 . These tiles are shown to tessellate by Lemma 2.4.

Class $\mathcal{T}_{2}$. Let $T$ be any $(n, q)$-gon belonging to this class. Then we observe that $n$ must be a multiple of 4 and that $\partial T$ is alternating in 00 and 11 . So we will verify that if $T$ is a tile with the sequence of markings on $\partial T$ of the form $00110011 \ldots 11$, then $T$ tessellates. To do this, we give a tiling algorithm.

Before giving the algorithm, we make an observation about this class of tiles that will figure heavily in our algorithm: if $T$ is in the class of tiles $\mathcal{T}_{2}$, then two edges away in either direction from an edge marked 0 is an edge marked 1 and vice versa.

To form a first corona, at edges marked 0 on $\partial T$ place a copy of $T$ using the triple 110 so that the 1 in the middle of this triple meets the edge marked 0 on $\partial T$, and at edges marked 1, place a copy of $T$ using the triple 100 so that the 0 in the middle of this triple meets the edge marked 1 on $\partial T$. This completes a first corona.

We proceed inductively. Suppose that a coronal configuration $\mathcal{C}_{k}$ consisting of $k$ coronas has been formed. To form the next coronal configuration, $\mathcal{C}_{k+1}$, we examine what is happening around the valence- 3 vertices on $\partial \mathcal{C}_{k}$ : Let $\mathcal{C}_{k-1}$ be the cononal configuration that is contained in $\mathcal{C}_{k}$ that is just $\mathcal{C}_{k}$ minus the outer corona, then consider an edge on $\partial \mathcal{C}_{k-1}$ that is incident to two valence- 2 vertices. If that edge is marked 1 , then the situation (up to reflection) depicted at the left in Fig. 11 is forced. Likewise, if the edge is marked 0 , then the situation (up to reflection) depicted at the right in Fig. 11 is forced. In either case, focusing now on the pair of "neighboring" valence- 3 vertices on $\partial \mathcal{C}_{k}$, notice that the outer edges incident to the valence- 3 vertices on $\partial \mathcal{C}_{k}$ have opposite parity. Similarly, the markings on edges incident to valence- 3 vertices on $\partial \mathcal{C}_{k-1}$ give rise to edges incident to neighboring pairs of valence- 3 vertices on $\partial \mathcal{C}_{k}$ such that the outer edges incident to these valence-3 vertices have opposite parity (see Fig. 12).

Now that it is established that the outer edges incident to neighboring valence-3 vertices on $\partial \mathcal{C}_{k}$ have opposite parity, we show how to complete the next corona. First place tiles at the valence- 3 vertices on $\partial \mathcal{C}_{k}$, as in Fig. 13. Next, fill in the tiles to cover the exposed edges between the valence-3 vertices as in Fig. 14 (using 011 and 001 as necessary). This completes this case.
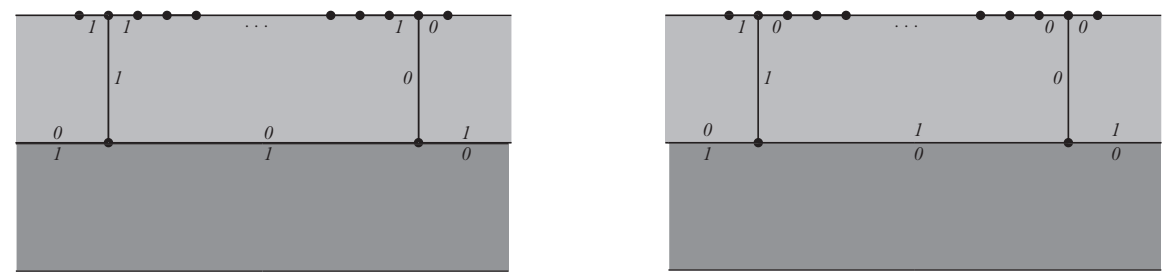

Fig. 11. The bottom tiles (shaded darker) are boundary tiles of $\mathcal{C}_{k-1}$ and the upper tiles (shaded lighter) are boundary tiles of $\partial \mathcal{C}_{k}$. The outer edges incident to the valence- 3 vertices on $\partial \mathcal{C}_{k}$ must have opposite parity. 

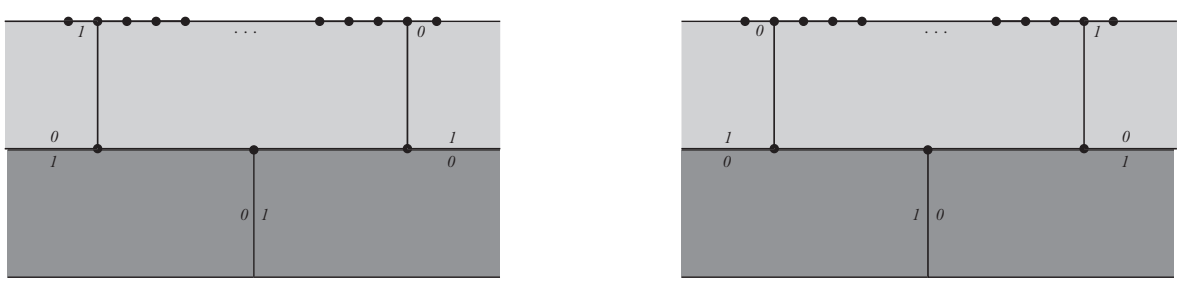

Fig. 12. The bottom tiles are boundary tiles of $\mathcal{C}_{k-1}$ and the upper tiles are boundary tiles of $\mathcal{C}_{k}$. The outer edges incident to the valence- 3 vertices on $\partial \mathcal{C}_{k}$ must have opposite parity.

Classes $\mathcal{T}_{3}$ and $\mathcal{T}_{6}$. Let $T$ be in any one of these families of tiles. We will find it convenient to consider the cases that $\partial T$ contains three, four, and at least five edges marked 0 .

Subcase 1: There are five or more edges marked 0 in $\partial T$. To form a first corona, at edges marked 0 place a copy of $T$ using the triple 110 so that the 1 in the middle of this triple meets the edge marked 0 on $\partial T$, and at edges marked 1, place a copy of $T$ using the triple 100 so that the 0 in the middle of this triple meets the edge marked 1 on $\partial T$. This completes a first corona.

Now we inductively show how to complete a tessellation. Suppose that a coronal configuration $\mathcal{C}$ is given. Begin the process of completing the next corona by placing copies of $T$ at the vertices on $\partial \mathcal{C}$ with valence 3 . The copies of $T$ that are placed at these valence- 3 vertices are forced to meet two copies of $T$ simultaneously, but this can always be done since the pairs 11, 01, 10, and 00 are all contained in $\partial T$. After placing these tiles at the valence- 3 vertices, we are left with the task of filling the tiles in between, as depicted in Fig. 15. We do not necessarily know the parity of the edges exposed on the right and left of the tiles just placed (labelled $y$ and $z$ in Fig. 15) at the valence-3 vertices, but it does not matter-we will show that all possibilities can be filled.

As in Fig. 15, label the edges of $\partial \mathcal{C}$ left exposed between any two of the just placed tiles at the valence- 3 vertices by $x_{1}, x_{2}, \ldots, x_{k}$. Note that these edges all belong to a single copy of $T$, so $k=n-6$ or $k=n-5$, depending on whether or not this copy of $T$ was placed incident to a valence- 3 or a valence- 2 vertex on the boundary of the previous corona. Either way, at least one of the edges labeled $x_{1}, x_{2}, \ldots, x_{k}$ must be marked 0 since in this subcase there are at least five edges marked 0 and not more than two consecutive edges marked 0 may be consecutive. Call this edge $x_{i}$.

Referring again to Fig. 15, there are only four possibilities for the markings of the edges labeled $y$ and $z$. In Figs. 16-19 we show how to fill in the tiles. We heavily use the fact that if $T$ is a member of either of the families $\mathcal{T}_{3}$ or $\mathcal{T}_{6}$, all of the following triples are present in $\partial T: 111,110,011,010,001$, and 100 (using a reflected copy of $T$ if needed). If $y$ and $z$ have opposite parity, we see that it is easy to fill in the tiles as in Figs. 16 and

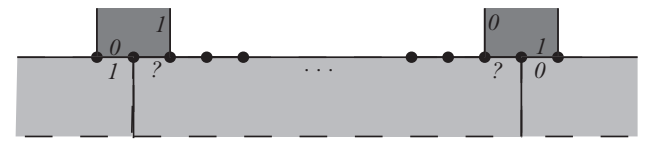

Fig. 13. How to place tiles at valence- 3 vertices on $\partial \mathcal{C}_{k}$. 


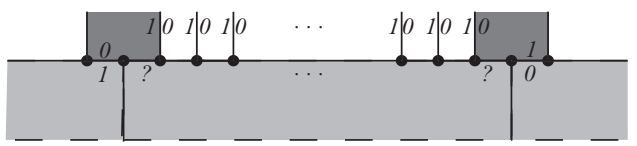

Fig. 14. How to fill in tiles between the tiles placed at valence- 3 vertices on $\partial \mathcal{C}_{k}$.

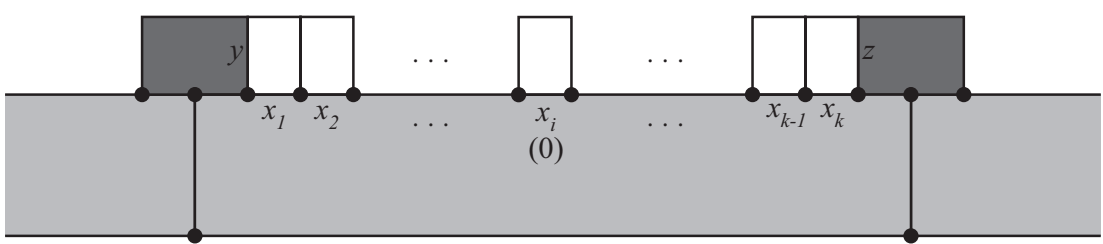

Fig. 15. The bottom tiles are boundary tiles of $\mathcal{C}$. To complete the next corona, tiles are first placed at valence-3 vertices on $\partial \mathcal{C}$. Next, tiles are placed along edges $x_{1}, \ldots, x_{k}$.

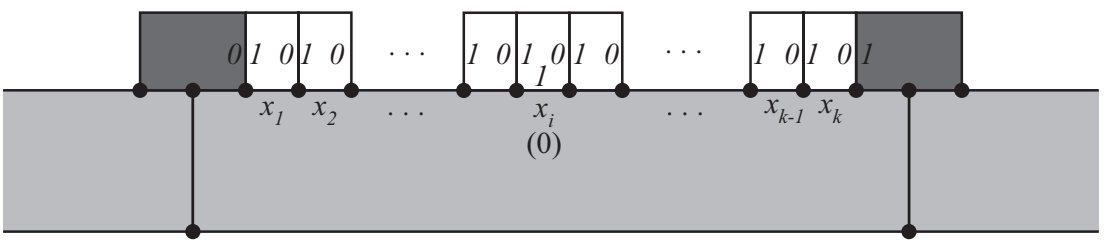

Fig. 16

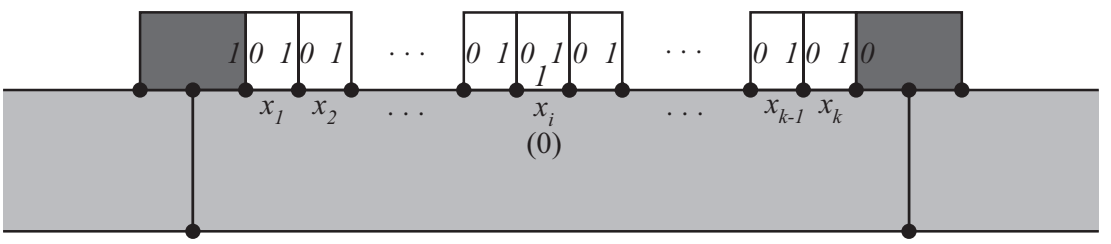

Fig. 17

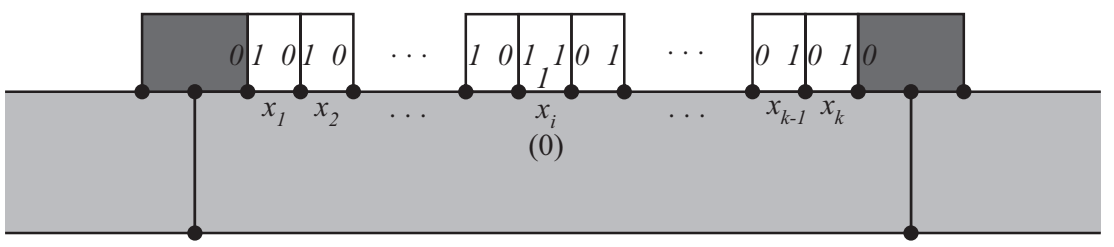

Fig. 18

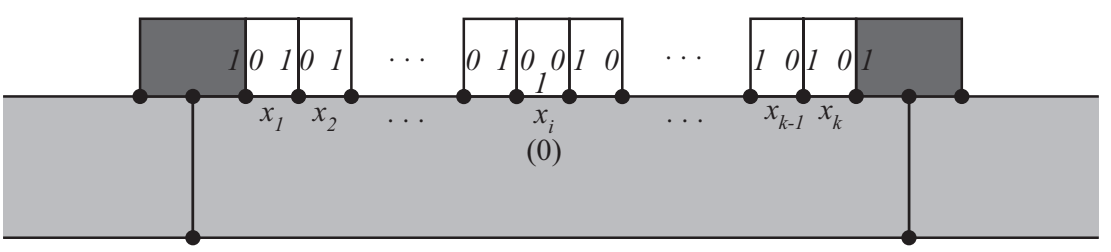

Fig. 19 
17. However, if $y$ and $z$ have the same parity, then we use the edge labeled $x_{i}$ that is marked 0 (along with the edge combinations 111 or 010 ) to merge the colliding flows, as in Figs. 18 and 19.

Subcase 2: There are only three edges marked 0 in $\partial T$. If there are only three edges marked 0 in $\partial T$, then $T$ cannot be a member of the family $\mathcal{T}_{3}$. The only possible form that $T$ may take in $\mathcal{T}_{6}$ is where

$$
\partial T=111 \cdots 10100 .
$$

Tiles of this form are shown to tessellate in Appendix A.

Subcase 3: There are only four edges marked 0 in $\partial T$. If $T$ is a member of the family $\mathcal{T}_{3}$ and $\partial T$ contains only four 0 's, then we must have

$$
\partial T=111 \cdots 100100,
$$

which we show tiles in Appendix B. If $T$ is a member of the family $\mathcal{T}_{6}$ and $\partial T$ only contains four 0's, then we must have

$$
\partial T=\underbrace{111 \cdots 1}_{m \geq 3} 00 \underbrace{111 \cdots 1}_{n \geq 1} 010
$$

or

$$
\partial T=\underbrace{111 \cdots 1}_{m \geq 3} 0 \underbrace{111 \cdots 1}_{n \geq 1} 0010 .
$$

Tiles of these last two forms can be shown to tessellate using a slightly modified version of the algorithm from Subcase 1. This is shown in Appendix C.

Class $\mathcal{T}_{4}$. Let $T$ be a member of $\mathcal{T}_{4}$. Then $\partial T$ contains 000 and 111. Either 000 or 111 are consecutive in $\partial T$ or not. These two possibilities form two subcases:

Subcase 1: 000 and 111 are consecutive in $\partial T$. Let $T$ be any such tile. We give a tiling algorithm:

Step 1. Form the first corona in such a way that the edges incident to the valence-3 vertices on the boundary of the first corona are marked 10 as at the left in Fig. 20.

Step 2. The second corona can be formed by filling in the tiles between the valence3 vertices on the boundary of the first corona as shown at the right in Fig. 20 while ensuring that the edges incident to the valence- 3 vertices appearing on the boundary of the second corona are marked 10 . So this procedure can be used inductively to complete a tessellation.

Subcase 2: 000 and 111 are not consecutive in $\partial T$. Let $T$ be any such tile. We give another tiling algorithm:

Step 1. First, note that $\partial T$ must contain the edge combinations 11100 and 11000. Form a first corona in the following manner: For each edge on $\partial T$ marked 0 , place a 


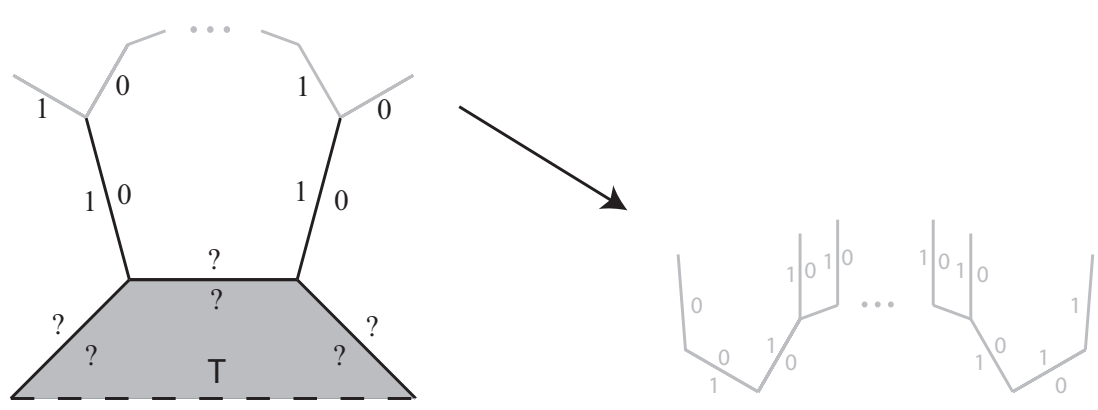

Fig. 20. Forming coronas for tiles containing 000 and 111.

copy of $T$ abutting this edge using the edge combination 11100 where the 1 in the middle of this combination meets the 0 on $\partial T$. Likewise, for each edge marked 1 on $\partial T$, place a copy of $T$ abutting this edge using the edge combination 11000 where the 0 in the middle of this combination meets the 1 on $\partial T$. In this way a first corona is formed. The edge to the left of each valence- 3 vertex on the boundary of the first corona is marked 0 and the edge to the right of each valence-3 vertex is marked 1.

Step 2. Suppose that a configuration $\mathcal{C}_{k}$ consisting of $k$ coronas has been formed. We may assume inductively that the edge to the left of each valence- 3 vertex on the boundary of $\mathcal{C}_{k}$ is marked 0 . In Fig. 21 we show that this inductive assumption implies that the edge to the left of each valence- 3 vertex on $\partial \mathcal{C}_{k+1}$ must be marked 0 . Figure 21 also shows how to place tiles at both valence- 2 and valence- 3 vertices on $\partial \mathcal{C}_{k}$, and these choices are compatible with one another since they set up a " 01 " flow around the corona.

Class $\mathcal{T}_{5}$. There is only one (6,3)-gon belonging to this class-it is marked 110010 . To show that this tile tessellates $\mathbb{E}^{2}$, we simply demonstrate a fundamental domain made from copies of this tile (Fig. 22). We note that there are no (7, 3)-gons in this class, but in a similar manner as with $(6,3)$-gons, we can establish fundamental domains for the possible $(8,3)$-gons (there are only two-11001010 and 11010010).

It remains to be shown that all $(n, 3)$-gons in this class with $n \geq 9$ tessellate $\mathbb{H}^{2}$. So let $T$ be any such tile. First, we form a single corona by doing the following: adjacent
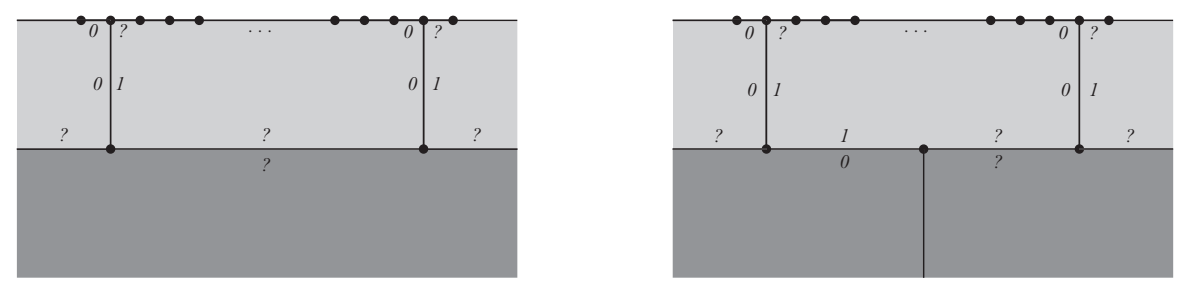

Fig. 21. Forming coronas for tiles containing nonconsecutive 000: the bottom tiles are the boundary tiles of the $k$ th corona and the top tiles are boundary tiles of the $(k+1)$ st corona. 


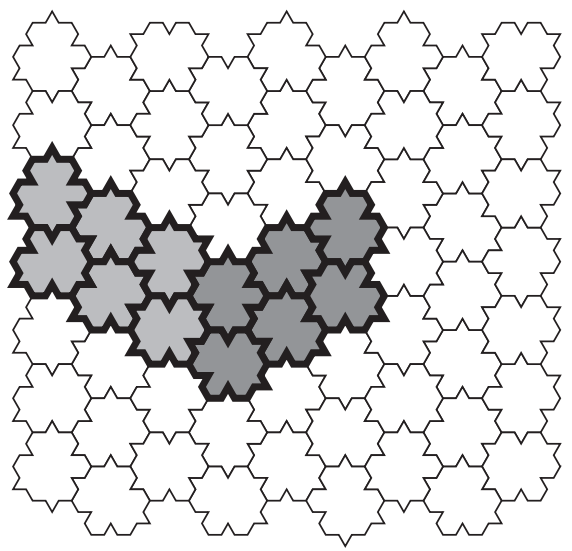

Fig. 22. One way to tessellate $\mathbb{E}^{2}$ using a (6, 3)-gon marked 110010. The fundamental domain is shaded.

to edges marked 1 on $\partial T$, place a copy of $T$ using the edge combination 001 so that the middle 0 meets the 1 on $\partial T$, and adjacent to edges marked 0 , place a copy of $T$ using the edge combination 011 so that the middle 1 meets the 0 on $\partial T$.

To complete the $(k+1)$ st corona, begin by placing tiles incident to the valence-3 vertices on the boundary - the particular orientations of these tiles just placed does not matter. On the boundary of the $k$ th corona, between these just placed tiles, there must be at least one 1 and one 0 . Thus colliding flows, as in the algorithm of Cases $\mathcal{T}_{3}$ and $\mathcal{T}_{6}$, may be merged (as in Fig. 15-17).

Class 77 . Let $T$ be a tile in this class of tiles. We observe that $\partial T$ must contain at least five edges marked 0 . If there are exactly five edges marked 0 in $\partial T$, then $T$ must be of the form

$$
111 \cdots 1000100
$$

Any tile of this form is shown to tessellate by the algorithm for Case $\mathcal{T}_{4}$, Subcase 1 . If $\partial T$ contains six or more edges marked 0 , then $T$ is shown to tessellate by the algorithm of Cases $\mathcal{T}_{3}$ and $\mathcal{T}_{6}$, Subcase 1 .

Class $\mathcal{T}_{8}$. In the construction of coronal configurations, it is never necessary for a tile just being placed to meet more than three tiles at once. This makes it clear that any tile in the family $\mathcal{T}_{8}$ tessellates since such a tile contains every triple of 0 and 1.

Classes $\mathcal{N}_{1}$ and $\mathcal{N}_{3}$. If $T$ belongs to this class, then on $\partial T$, two edges away from an edge marked 0 (in either direction) is an edge marked 1 . Now consider the sequence of edges on $\partial T$ labeled 111: placing tiles adjacent to these edges (keeping in mind our previous observation) forces a configuration combinatorially equivalent to the diagram at the left in Fig. 23.

We then see that a second corona cannot be formed around this part of the first corona as edges with the same parity will be forced to meet (this observation heavily uses the fact that on $\partial T$, two edges away from an edge marked 0 is an edge marked 1). 

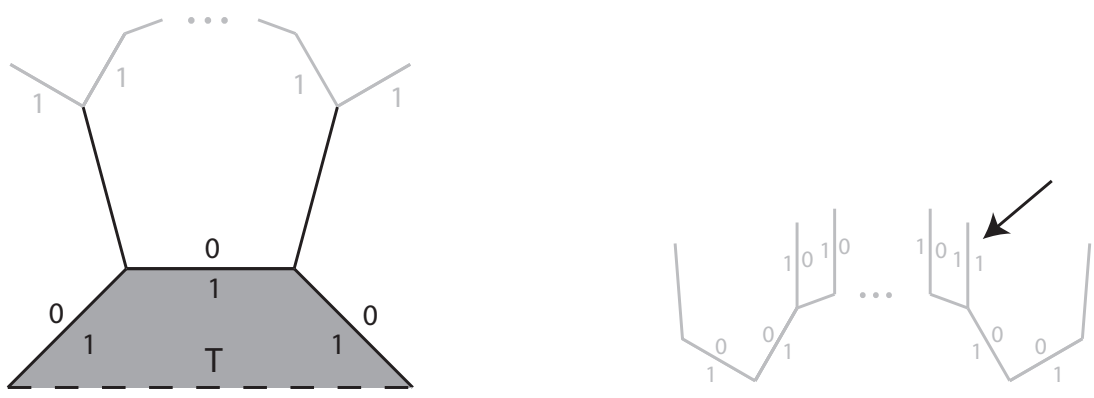

Fig. 23. (Left) We form the part of the first corona extending from the edges marked 111 on $\partial T$. (Right) We see than we cannot extend the part of the first corona from the left diagram since two edges labeled 1 will be forced to meet.

Class $\mathcal{N}_{2}$. This case is very similar to the previous case. Suppose $T$ belongs to class $\mathcal{N}_{2}$. In this case, as in the previous case, we observe that on $\partial T$, two edges away from an edge marked 0 (in either direction) is an edge marked 1 . Using a slightly modified version of Fig. 23, we can see that it is not possible to form the section of the second corona extending from the sequence of edges on $\partial T$ labeled 101: as in Case $\mathcal{N}_{1}$, edges with equal parity will be forced to meet.

For classes $\mathcal{N}_{1}, \mathcal{N}_{2}$, and $\mathcal{N}_{3}$, we see that a first corona can be formed each time. Indeed, if $T$ is an (n,3)-gon in any class containing 001 and 011, then $H(T) \geq 1$. Classes $\mathcal{N}_{1}$, $\mathcal{N}_{2}$, and $\mathcal{N}_{3}$ contain 001 and 011 , but since we proved that second coronas cannot be formed in each of these cases, then any tile $T$ in classes $\mathcal{N}_{1}, \mathcal{N}_{2}$, and $\mathcal{N}_{3}$ has $H(T)=1$. For example, the (7, 3)-gon of Fig. 24 is in class $\mathcal{N}_{1}$ and has Heesch number 1.

\section{Future Work}

In the theorems proven here, it is required that every edge be marked with either a bump or a nick. Historically, however, tiles admitting some unmarked edges have made for
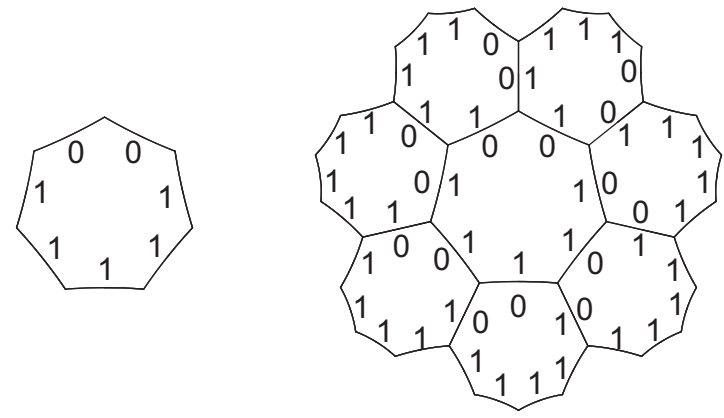

Fig. 24. A (7, 3)-gon with Heesch number 1. 
interesting examples as well, such as Ammann's Heesch number 3 hexagon (Fig. 3). This brings up the following problem:

Unsolved Problem 4.1. Which regular polygons, each of whose edges is either unmarked or marked with either a bump or a nick, tessellate? Additionally, what is the maximum finite Heesch number allowed by the nontessellating tiles (if such a maximum exists)?

It is also interesting to consider different kinds of edge markings, such as colored edges (we require that edges of the same color may meet) and directed edges. Some work has begun in this direction. In an undergraduate research project, Bobby Thomas has begun the complete classification of Euclidean hexagons, each of whose edges is marked with a combination of bumps/nicks, colors, and directions. This work can also be generalized to $\mathbb{H}^{2}$ and to tiles formed from fusing multiple polygons together. This brings up the following unsolved problem:

Unsolved Problem 4.2. Which regular polygons, each of whose edges is marked with a combination of bumps or nicks, colors, and/or directions, tessellate the plane ( $\mathbb{H}^{2}$ or $\mathbb{E}^{2}$ )? Additionally, what is the mazimum finite Heesch number allowed by these tiles (if any)?

\section{Acknowledgements}

The author thanks Dr. Chaim Goodman-Strauss for his guidance and help with this article, and he also thanks the referees for their helpful suggestions.

\section{Appendix A. $\quad(n, 3)$-Gons of the Form $111 \cdots 10100$}

To form a first corona, at edges marked 0 on $\partial T$ place a second copy of $T$ using the triple 110 so that the 1 in the middle of this triple meets the edge marked 0 on the first tile, and at edges marked 1 on $\partial T$ place a copy of $T$ using the triple 100 so that the 0 in the middle of this triple meets the edge marked 1 on the first tile.

To form subsequent coronas, we begin by placing tiles at the valence- 3 vertices on the boundary; for the algorithm of this subcase, we restrict ourselves to certain choices for placing these tiles at valence- 3 boundary vertices. Before describing what these choices will be, we examine the types of valence- 3 vertices that may appear on the boundary. On the boundary of coronal configurations of size 2 or more, there are two different types of valence- 3 vertices:

- We define a Type I valence-3 vertex on the boundary to be a valence-3 vertex that is contributed by a tile that is incident to a valence- 3 vertex on the boundary of the previous corona (see Fig. 25). Note that these Type I valence-3 vertices come in pairs (two for each valence-3 vertex in the previous corona). 


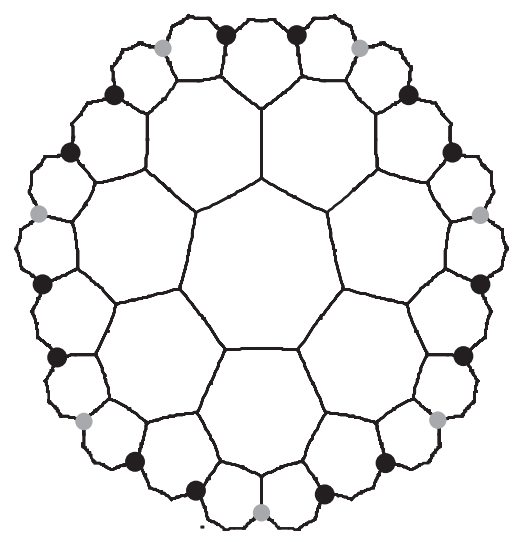

Fig. 25. Type I vertices are black and Type II vertices are grey.

- A Type II valence-3 vertex on the boundary is a vertex that is not contributed by a tile that is incident to a valence- 3 vertex on the boundary of the previous corona.

Next we give the tiling algorithm for this case. First, we specify that tiles placed at valence-3 vertices of either type must be placed in one of the eight ways shown in Fig. 26. Using these choices, notice that the interior boundary edges incident to the resulting Type I valence-3 vertices are never both marked with a 1. Thus, given a configuration of $k$ coronas formed using these choices at the Type I vertices, we can inductively assume that the interior boundary edges incident to the Type I valence- 3 vertices on the boundary of any coronal configuration are never both marked 1 .

Step 1 . We proceed inductively. Suppose a coronal configuration of $k$ coronas is given that was formed using the rules for placement of valence-3 vertices given above (trivially one can check that a second corona can be formed in this way). To form the $(k+1)$ st corona, we begin by placing tiles at the Type I valence- 3 vertices. In particular, we are interested in the pairs of Type I vertices that are "spawned" from the same valence-3 vertex in the previous corona. Between these vertices there are several edges belonging to a single copy of the tile, and we want our choice of tile placement at the pairs of Type I vertices to be done in such a way as to make for easy placement of the tiles along

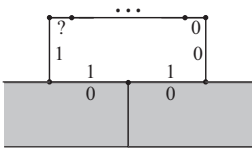

$-\mathrm{OR}$ -

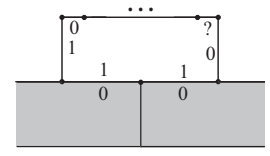

Fig. 26. How tiles are to be placed at valence- 3 vertices. The shaded tiles are boundary tiles of a previously formed corona.

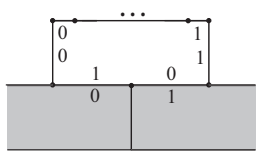

- OR -
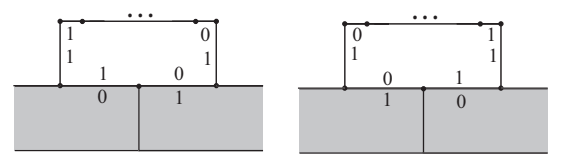
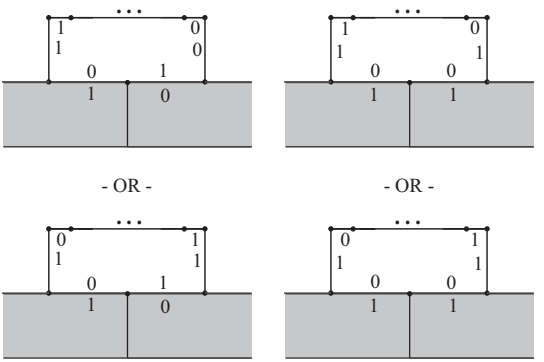

- OR - 


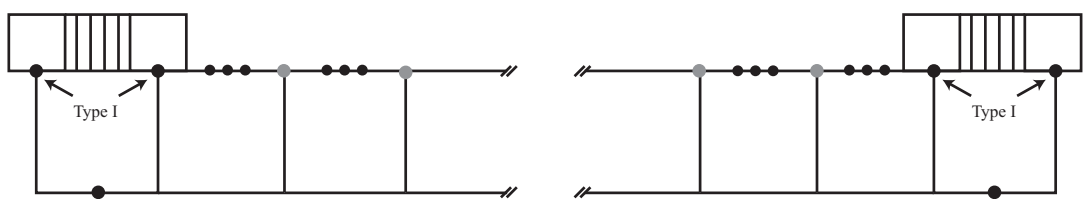

Fig. 27. A schematic of a portion of the boundary configuration after tiles are placed at and between pairs of Type I valence-3 vertices.

these edges between a given pair of Type I vertices. Just as in the algorithm for the more general case, if one of the edges between but not incident to these Type I valence-3 vertices is marked 0 , then we can place tiles at these edges without regard for how tiles are placed at the Type I vertices (ala Figs. 16-19). However, in this subcase we have only three edges marked 0 , so we are not guaranteed that there will be a boundary edge marked 0 between but not incident to these pairs of Type I vertices. Therefore we must be careful how we choose to place the tiles at these vertices. Similarly to what we did in the Cases $\mathcal{T}_{3}$ and $\mathcal{T}_{6}$ (the current case is a subcase of Case $\mathcal{T}_{6}$ ) depicted in Figs. 16 and 17, we make sure that the inwardly facing edges of the tiles placed at the Type I vertices have opposite parity. Keeping in mind our inductive hypothesis that the interior boundary edges incident to the resulting Type I valence- 3 vertices are never both marked with a 1 , we see that there are 16 possible combinations of markings for these edges. A quick check of these 16 possible combinations of boundary edges at the pairs of Type I shows that we can always arrange it so that the inwardly facing edges will have opposite parity, and so we fill in the tiles between in a way similar to that as shown in Figs. 16 and 17 (the edge marked 0 in the middle is not required since the inwardly facing edges have opposite parity). To make it easier to form the rest of the corona, we further stipulate that when we place a tile at a right-side Type I valence-3 vertex whose incident boundary edges are marked 10 we use the first option from Fig. 26, and when we place a tile at a left-side Type I valence- 3 vertex whose incident boundary edges are marked 01 we use the second option from Fig. 26.

Step 2. At this point, a representative portion the boundary of the current configuration is depicted schematically in Fig. 27. Next we fill in the remaining slots that are not incident to or between pairs of Type I valence- 3 vertices. This can be accomplished because between most pairs of the remaining Type II vertices there must be marked a 0 that is not incident to the Type II vertices that we can use along with the edge combination 010 to merge tiles, regardless of how tiles are placed at the Type II valence- 3 vertices. ${ }^{3}$ The only exception to this is when the tile of the previous corona that contributes the edges between a pair of Type II vertices meets the tile to the left, the tile in the previous corona, and the tile to the right with the edge combination 010. This exception can be handled with ease by reflecting that tile if necessary to make sure the facing edges of the tiles placed at the Type II vertices are of opposite parity.

\footnotetext{
${ }^{3}$ It may be that we must change the orientation of the tiles in the previous corona slightly to ensure that such a 0 must appear between each pair of Type II vertices, but that change can be performed without disturbing any of the surrounding tiles, so we might as well assume that it has already been done. These edges marked 0 serve to merge surrounding tiles, regardless of the placement at the Type I vertices.
} 

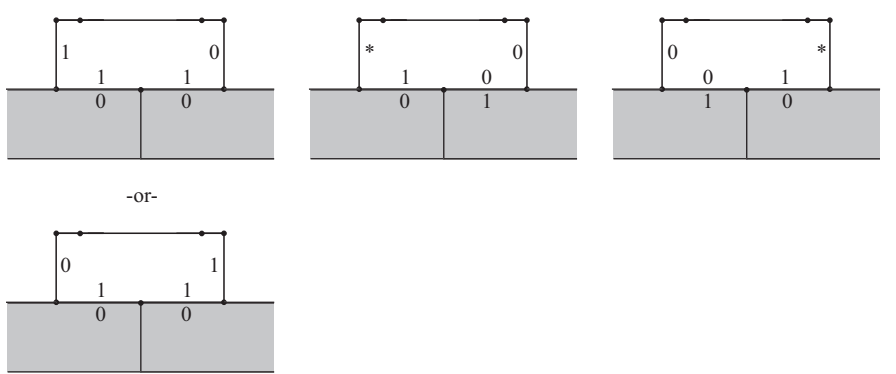

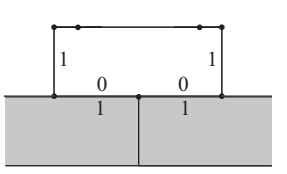

Fig. 28. Placement of tiles at valence- 3 vertices for tiles of the form $111 \cdots 100100$. Here $*$ can be either 0 or 1 as needed.

\section{Appendix B. $\quad(n, 3)$-Gons of the Form $111 \cdots 100100$}

Begin by forming a first corona in the manner we have described previously. To form successive coronas, we first place tiles at the valence-3 vertices as specified in Fig. 28. After placing tiles at the valence- 3 vertices, we fill in the remaining tiles to form the corona; indeed, the choice of placement at the valence- 3 vertices is made in such a way to ensure that these remaining slots can be filled. The remainder of this section describes in detail this process and justifies why it works.

The main observation for this case is that with our choices for placing tiles at valence-3 vertices as in Fig. 28, one of two things will occur with each pair of valence-3 boundary vertices: (1) the inside edges of the tiles placed at the valence- 3 vertices will have opposite parity, in which case it is easy to fill in the slots between, or (2) if the inside edges of the tiles placed at the valence- 3 vertices do not have opposite parity, then one of the edges between but not incident to the valence- 3 vertices will be marked 0 , which allows for the easy placement of tiles into the remaining slots. To see this, we use the notation $a b \cdots c d$ to denote the boundary configuration depicted in Fig. 29. Using this notation, the 16 possible combinations are $11 \cdots 11,11 \cdots 10,11 \cdots 01,11 \cdots 00,10 \cdots 11,10 \cdots 10,10 \cdots 01$, $10 \cdots 00,01 \cdots 11,01 \cdots 10,01 \cdots 01,01 \cdots 00,00 \cdots 11,00 \cdots 10,00 \cdots 01$, and $00 \ldots 00$. There are only a few cases where using the placement rules in Figure 28 do not satisfy (1) above: these are $11 \cdots 11,01 \cdots 10$, and potentially any case involving 00. We handle these cases individually:

Cases $11 \cdots 11$ and $01 \cdots 10$. Notice that the inside boundary edges adjacent to the valence- 3 vertices are both marked 1 . This means that one of the edges between but not incident to the valence- 3 boundary vertices must be marked 0 because all four of the edges marked 0 cannot possibly be meeting other tiles in the current or previous corona-at least one is exposed on the boundary. This 0 can be used to merge whatever tile placements are required using the edge combinations 010 or 111.

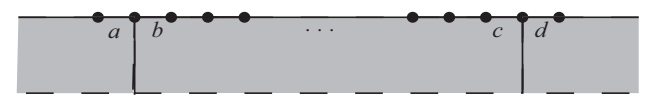

Fig. 29. The boundary configuration $a b \cdots c d$. 
Cases involving 00 . The key observation here is that at valence- 3 vertices whose incident boundary edges are marked 00 , there must be another edge marked 0 , either immediately to the left of the leftmost 0 in the pair 00 or immediately to the right of the rightmost 0 in the pair 00 . Call this edge marked 0 a free 0 . After placing a tile at valence3 incident edges marked 00 , the edges facing left or right must have opposite parity, but we have two choices for the orientation of this tile, so we use whichever marking we need on the side opposite the free 0 so that the facing edges have opposite parity. On the side of the free 0 the marking on the facing edges does not matter-the free 0 can be used to merge whatever tile placements are required using the edge combinations 010 or 111.

\section{Appendix C. $\quad(n, 3)$-Gons of the Form $111 \cdots 100111 \cdots 1010$ or}

$$
111 \cdots 10111 \cdots 10010
$$

Let $T$ be a tile in either of the families of tiles whose boundary edges are marked either

$$
\underbrace{111 \cdots 1}_{m \geq 3} 00 \underbrace{111 \cdots 1}_{n \geq 1} 010
$$

or

$$
\underbrace{111 \cdots 1}_{m \geq 3} 0 \underbrace{111 \cdots 1}_{n \geq 1} 0010 .
$$

If $n>1$, we can use the same algorithm as we used for Subcase 1 of $\mathcal{T}_{3}$ and $\mathcal{T}_{6}$, as there will necessarily be a 0 on the coronal boundary as in Fig. 15 .

If $n=1$, we use a slightly modified version of the proof for Cases $\mathcal{T}_{3}$ and $\mathcal{T}_{6}$, Subcase 1. There we considered a sequence of edges $x_{1}, x_{2}, \ldots, x_{k}$ on $\partial \mathcal{C}$ (see Fig. 15). For the family of tiles under consideration here, we cannot guarantee that one of these edges is marked 0 as we could previously. However, in this case we can make the following observation: if $x_{i}$ is marked 1 for every $i, 1 \leq i \leq k$, then the edge on $\partial \mathcal{C}$ immediately to the left of $x_{1}$ is marked 0 and the edge immediately to the right of $x_{k}$ is also marked 0 . Again referring to Fig. 15, we can now place tiles at the valence- 3 vertices on $\partial \mathcal{C}$ so that the edge labeled $y$ is marked 0 and the edge labeled $x$ is marked 1 . This can be done no matter what combination of two edges is need to place tiles at the valence- 3 vertices on $\partial \mathcal{C}$ since the edge combinations 010,110 , and 111 are all contained in $\partial T$. Also, this manner of tile placement at the valence- 3 vertices on $\partial \mathcal{C}$ is compatible with whatever arrangement of tiles may lay to the left and right of the valence- 3 vertices.

\section{References}

1. R. Berger, The Undecidability of the Domino Problem, Memoirs of the American Mathematical Society, No. 66, AMS, Providence, RI, 1966.

2. C. Goodman-Strauss, Open questions in tilings, submitted.

3. G. Grünbaum and G. C. Shephard, Tilings and Patterns, Freeman, New York, 1987.

4. C. Mann, Heesch's Problem and Other Tiling problems, Ph.D. Dissertation, University of Arkansas at Fayetteville, 2001. 
5. C. Mann, Heesch's tiling problem, American Mathematical Monthly, 111 (2004), 509-517.

6. G. A. Margulis and S. Mozes, Aperiodic tilings of the hyperbolic plane by convex polygons, Israel Journal of Mathematics, 107 (1998), 319-325.

7. R. Penrose, Pentaplexity, Mathematical Intellegencer, 2 (1979), 32-37.

8. R. M. Robinson, Undecidability and nonperiodicity of tilings of the plane, Inventiones Mathematicae, $\mathbf{1 2}$ (1971), 177-909.

9. S. Staul, The Poincaré Half-Plane: A Gateway to Modern Geometry, Jones and Bartlett, Boston, MA, 1993.

Received February 6, 2004, and in revised form September 9, 2004. Online publication March 18, 2005. 